\title{
Fasting induces FGF21 in humans
}

A new study shows that circulating levels of FGF21 are markedly increased by fasting in humans and are part of the late stages of the body's adaptive response to starvation-a protective mechanism that evolved to aid survival in periods of famine.

In contrast to mice, in which levels of FGF21 are rapidly increased by fasting, previous studies in humans have either demonstrated no increase or only a modest increase in levels of FGF21 in response to fasting. "We felt that a conclusive fasting study needed to be performed in healthy individuals to definitively answer whether FGF21 was a fasting-induced hormone in humans and to understand what happens to levels of FGF21 during fasting," explains joint-lead investigator Pouneh Fazeli.

The researchers serially measured levels of FGF21 in 11 healthy male and female volunteers (aged

22.4-48.3 years; BMI $22.7-29.3 \mathrm{~kg} / \mathrm{m}^{2}$ ) during a 10-day fast. Although decreased in the early phase of the fast, levels of FGF21 at day 10 were fourfold higher than those at day 0 . Induction of FGF21 was associated with decreased thermogenesis of brown adipose tissue (measured by PET/MRI) and reduced levels of adiponectin, and occurred after the ketogenic response. Furthermore, FGF21 induction closely correlated with the release of markers of tissue breakdown (serum transaminases) and with weight loss.

"Our study definitively establishes FGF21 as a fasting-induced hormone in humans," states co-lead investigator Matthew Steinhauser. "By measuring FGF21 levels serially over a full 10-day fast in healthy volunteers, we now know why previous studies in humans reported inconsistent results, namely that FGF21 is induced in the majority of individuals, but only after a long-term fast."

On the basis of beneficial metabolic effects elicited by FGF21 treatment in mice (prevention of diet-induced obesity and improved glucose tolerance), FGF21 mimetics are being developed to treat metabolic diseases such as type 2 diabetes mellitus. "Our finding that the function of FGF21 might be highly dependent on an individual's nutritional status could be an important consideration as these potential drugs are developed," cautions Steinhauser.

David Holmes

ORIGINAL ARTICLE Fazeli, P. K. et al. FGF21 and the late adaptive response to starvation in humans. J. Clin. Invest. http://dx.doi.org/10.1172/ JCl83349 